\title{
Locked Nuclear Quadrupole Resonance Spectrometer for Pressure Measurements
}

\author{
Ronald C. Frisch and David L. VanderHart \\ Institute for Materials Research, National Bureau of Standards, Washington, D. C. 20234
}

(May 18, 1970)

\begin{abstract}
The Nuclear Quadrupole Resonance frequency of a nucleus in a solid is dependent on its local environment and can be quite sensitive to changes in temperature and pressure. A spectrometer capable of locking accurately to the center of a resonance signal is described. The feasibility of using the quadrupole resonance frequency as a transfer gage for precise pressure measurements is discussed using ${ }^{35} \mathrm{Cl}$ resonance in a $\mathrm{KClO}_{3}$ polycrystalline sample. The performance of the instrument implies a limiting accuracy for pressure measurements of 0.7 bar; preliminary results are presented showing frequency versus pressure curves near room temperature. Uncertainties of these measurements are primarily due to inadequate temperature control and the uncertainty of the pressure measurement.
\end{abstract}

Key words: $\mathrm{KClO}_{3}$ nuclear quadrupole resonance; pressure transducer; spectrometer.

\section{Introduction}

Many workers in the field of nuclear quadrupole resonance $(\mathrm{NQR})$ have for some time been interested in the temperature and pressure dependence of the NQR frequency. The first theoretical treatment was given by Bayer $[1]^{1}$ who considered temperature effects in terms of molecular vibrations. Kushida, Benedek, and Bloembergen [2] expanded the theory to take into effect thermal expansion and compressibility. Their results suggest that the NQR frequency might be useful as a sensing device for temperature, and in principle it could also be used to measure pressure. A material suitable for such experiments should be characterized by a strong resonance signal, a relatively narrow line width, and a lack of hysteresis. One 2 such material, which has been considered for an NQR thermometer $[2-6]$, is $\mathrm{KClO}_{3}$. In the early experiments, $\mathrm{NQR}$ spectrometers were not locked to the center of the resonance line and the experimental accuracy was of the order of $0.002 \mathrm{~K}$. Volpicelli et al. [7] have described a system for locking the spectrometer to the center of the resonance line. Most recently Utton [8] using a spectrometer locked to the center of the resonance line has been able to measure the temperature in the range 50 to $297 \mathrm{~K}$ to an accuracy of $\pm 0.001 \mathrm{~K}$.

The effects of pressure on the ${ }^{35} \mathrm{Cl} \mathrm{NQR}$ frequency in $\mathrm{KClO}_{3}[2,2 \mathrm{a}]$ are not as great as the temperature variations, i.e., $\left(\frac{\partial f}{\partial T}\right)_{P} \simeq 5 \mathrm{KHz} /{ }^{\circ} \mathrm{C}$ at room temperature

\footnotetext{
${ }^{1}$ Figures in brackets indicate the literature references at the end of this paper.
}

while $\left(\frac{\partial f}{\partial \bar{P}}\right)_{T} \simeq 30 \mathrm{~Hz} /$ bar. $^{2}$ Nevertheless, we decided to examine the feasibility of using the pressure dependence of the quadrupole resonance frequency as a method to measure pressure with high precision. If it could be shown that the resonance frequency is a stable, singlevalued function of both temperature and pressure, and that its dependence on contamination and crystallinity and other sources of error is below a certain value, then the quadrupole gauge would have one great advantage over other types of transfer gages: once the pressuretemperature dependence of the quadrupole frequency for a particular material had been established, any number of transfer gages could be reproduced and used within established limits of uncertainty without further need for calibration. In contrast all of the transfer gages presently available require initial calibration and frequent recalibration depending on their use.

It is clear from the nature of the application for which this spectrometer was designed, that it must be very stable; in addition, the sensitivity characteristics must be good because of the small sample size dictated by the geometry of the pressure vessel. This paper describes an improved version of the NQR tracking spectrometer used by Utton [8] for temperature studies.

With this system we have been able to track the ${ }^{35} \mathrm{Cl}$ NQR signal in $\mathrm{KClO}_{3}$ and measure the center frequency of the ${ }^{35} \mathrm{Cl}$ resonance to 1 percent of the line width ${ }^{3}$ for pressures up to $2 \mathrm{kbar}$. In constructing a calibration curve

\footnotetext{
${ }^{2} 1 \mathrm{bar}=10^{5} \mathrm{~N} / \mathrm{m}^{2}$.
} 


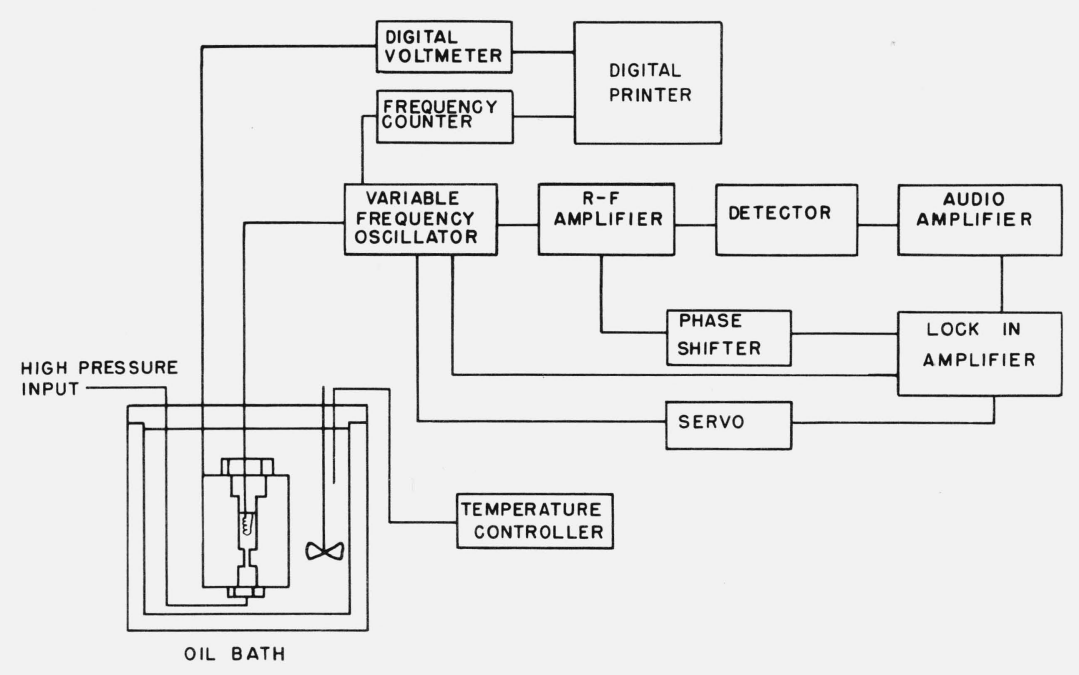

FIGURE 1. Block diagram of NQR Spectrometer.

the best pressure uncertainty in the range $0-2 \mathrm{kbar}$, is at present 0.15 bars at $2 \mathrm{kbars}$ and the percent error is about the same over the range [9]. The fluctuation in the temperature is reflected in the uncertainty of the frequency measurement at each pressure point and would correspond, based on the known temperature coefficient of $\mathrm{KClO}_{3}$, to about 0.25 bars when the sample temperature is held constant to $\pm 0.001{ }^{\circ} \mathrm{C}$. The uncertainty in the frequency counter is $\pm 1.0 \mathrm{~Hz}$. or about 0.03 bars. Hence the limiting uncertainty for determining the pressure from a frequency measurement, using such a calibration curve, would be about 0.7 bars.

\section{Instrumentation}

\subsection{Oscillator}

A block diagram of the quadrupole spectrometer which was used for the high pressure NQR studies is shown in figure 1. The oscillator detector circuit is given in figure 2 . The oscillator detector circuit is a modified version of the circuit described by Pound and Knight [10] and is essentially that used by Utton [8]. The oscillator is of the marginal type and oscillation is maintained by a nonlinear negative resistance provided by the tube feedback circuit [11]. The variable capacitors $C_{1}, C_{2}$ are used for coarse and fine tuning of the oscillator, and the voltage variable capacity diodes (VCD) are used for frequency tracking and frequency modulation. VCDl can also be used for tuning and will be discussed later. For various applications different values of $\mathrm{L}$ can be used to obtain any desired operating frequency. The $r$-f level at the sample coil can be varied by adjusting the gain control, $\mathrm{Rg}$, or by adjusting the feedback. At $28 \mathrm{MHz}$, for example, the $r-f$ level is adjustable over the range 0.02 $2.20 \mathrm{~V}$.

\subsection{R-F Amplifier}

The oscillator is followed by a three-stage stagger tuned

${ }^{3}$ Distance between first derivative extrema. r-f amplifier. By tuning each stage of an amplifier at slightly different frequencies it is possible to achieve a gain bandwidth product greater than that of synchronously tuned cascade stages. The selectivity curve can also be tailored to fit a prescribed response such as a flat band pass with very good gain [12]. The choice of a flat band pass $r-f$ amplifier is suggested by the operation of the oscillator detector circuit [10]. In the oscillator the level of oscillation is sensitive to the shunt impedance across the tank circuit. As the frequency is varied the corresponding change in the shunt impedance results in a change in the $r-f$ level. This undesirable change in level is compensated for by feeding back part of the $r-f$ rectified voltage in such a way as to control the value of the negative resistance, thereby holding the level of oscillation constant at a level far below natural limiting. In the case where the frequency of oscillation is expected to vary over a large range, as in a tracking spectrometer, it is desirable to have the voltage gain of the $r-f$ amplifier constant over the frequency range of operation. As a result the $d$-c feedback voltage, which controls the $r$ - $f$ level, is returned to the oscillator essentially independent of frequency within the band pass. The time constant for this feedback loop, of course, is long compared to the modulation frequency, which contains the signal information.

The $r-f$ amplifier used in this spectrometer was designed, using the prescription of Valley and Wallman [12], for a center frequency of $28 \mathrm{MHz}$ and $6 \mathrm{MHz}$ bandpass.

Knowing the bandwidth of each stage and the central frequency one can easily calculate the circuit component values. In figure 3 the a-c equivalent circuit of a typical stage is shown. Given the stage bandwidth, $B_{i}$, the loading resistor $R_{i}$ can be calculated from the relation $B_{i}=$ $\left(2 \pi R_{i} C_{i}\right)^{-1}$. The capacity $C_{i}$ is the sum of the output capacity of $T_{i}$ plus the input capacity of $T_{i+1}$ plus wiring capacity; and the tuning coil, $L_{i}$, is wound to resonate with $C_{i}$ at the appropriate center frequency. An oscilloscope trace of the band pass for this amplifier is shown in figure 4.

In a cascaded linear amplifier without feedback the 


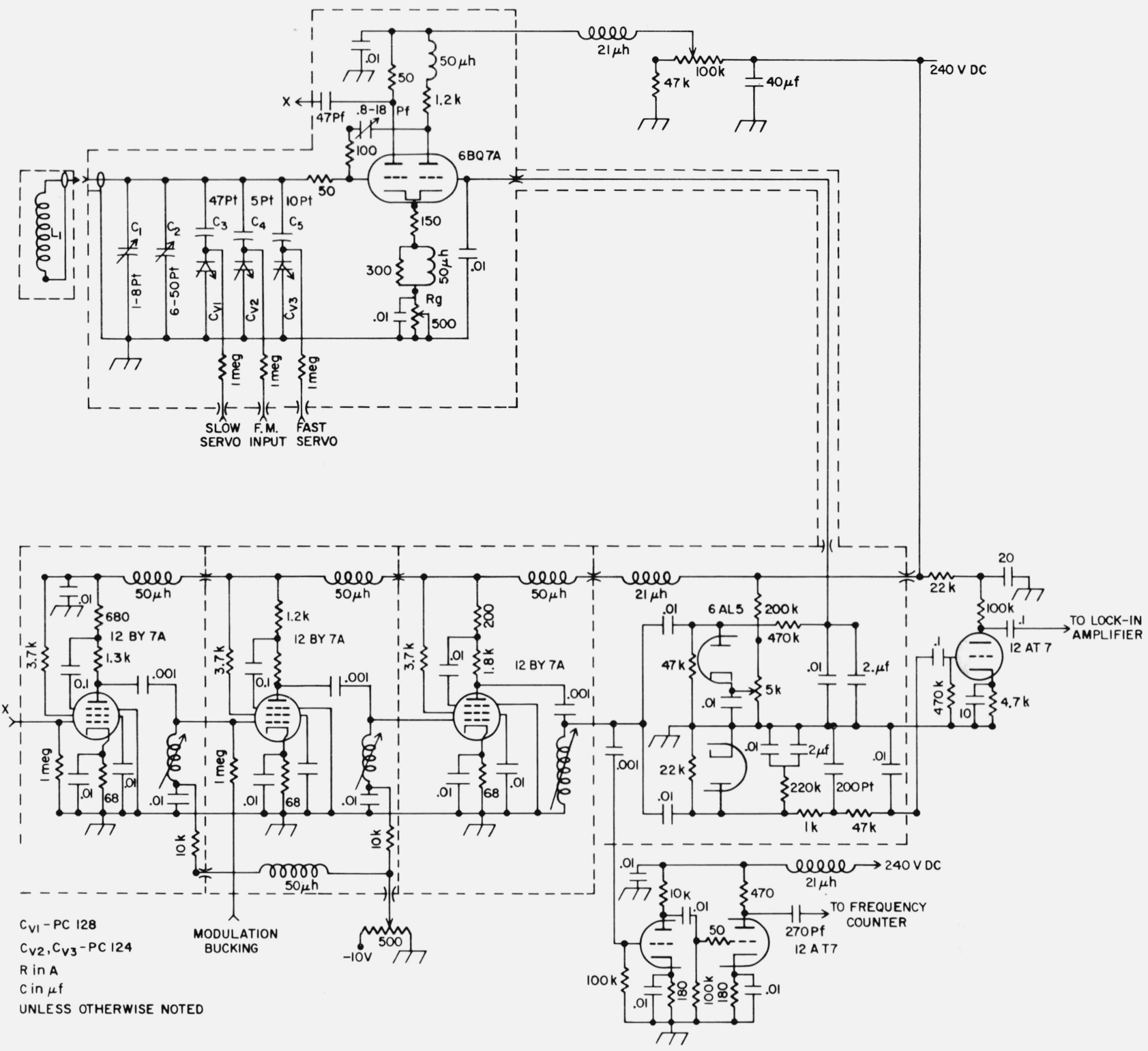

Figure 2. Oscillator detector circuit.

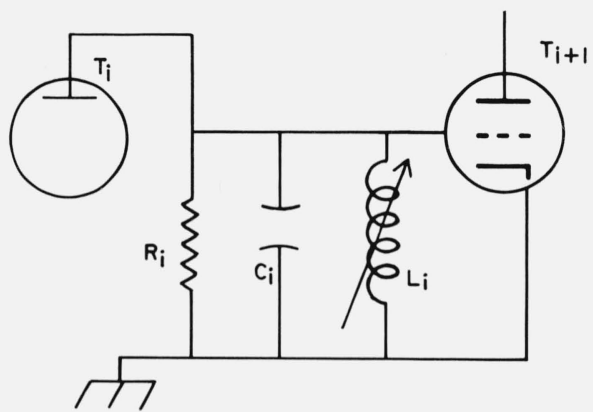

Figure 3. A-C equivalent circuit of a typical stage in rf amplifier.

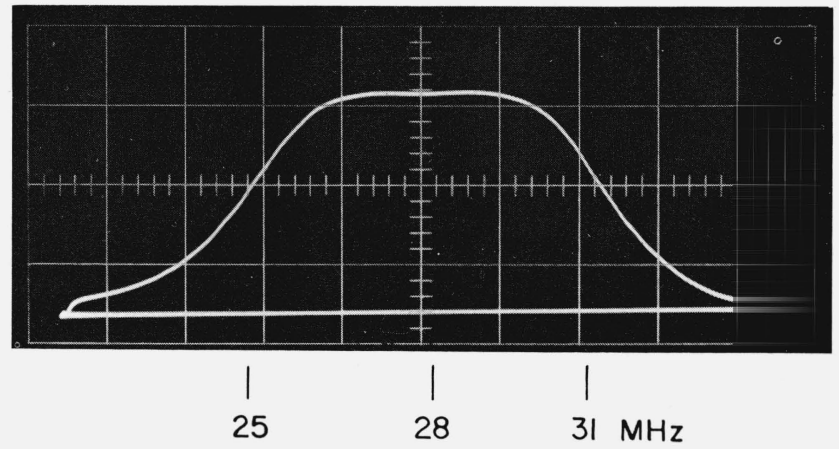

Figure 4. Oscilliscope trace of the band pass of the if amplifier. 
overall gain at any frequency, $f$, is the product of the individual stage gains at that frequency. The stage gain is given by

$$
G_{i}(f)=g_{m i} Z_{i}(f)
$$

where $Z_{i}(f)$ is the impedance function for a single stage. Because of this multiplicative property, if one of the stages has its $g_{m i}$ reduced but its selectivity characteristic, which is determined by $Z_{i}(f)$, left unchanged, the overall gain is reduced in proportion, whereas the overall selectivity curve is completely unaffected. Consequently, to the extent to which tube capacities and loading do not vary with $g_{m}$, it is possible to gain control a stagger-tuned amplifier in any stage or combination of stages [12]. In our case this is accomplished by adjusting the grid voltage on the first two stages.

Following the $r-f$ amplifier the signal is branched; one branch going to a buffer amplifier followed by an electronic frequency counter [13]; the other branch going to the diode detector. The detected signal is then fed into a lock-in amplifier whose output appears as the first derivative of an absorption curve. This is then used to provide the error signal for a servo mechanism which locks the oscillator frequency to the center of the resonance line.

The servo control system is similar to that described by Volpicelli et al. [7] and utilizes two control loops. The fast loop, which responds directly to the error signal and provides a quick response to short-term fluctuations, has a range up to $2 \mathrm{KHz}$. The second loop has a much slower response and utilizes a commercially available servo amplifier [14] and motor. Tachometer feedback is used and the motor is coupled to a 10-turn potentiometer via a gear box and antibacklash gears giving a total reduction ratio of $1000: 1$. By coupling the potentiometer shaft to the gear train with a dual magnetic clutch it is also possible to manually adjust the voltage on VCDI and hence the oscillator frequency, or with the aid of a second motor and gear drive, sweep the frequency. The ability to manually adiust the oscillator frequency greatly facilitates the initial locking of the servo system to the center of the resonance line.

Frequency modulation is provided by varying the voltage on VCD2 at the modulation frequency. As a result of the change in capacity in the tuned circuit the quality factor is also modulated which in turn leads to a modulation of the oscillation level. The result of this incidental amplitude modulation causes a signal to appear at the lock-in detector. The resulting shift in the base line of the $\mathrm{NQR}$ signal must be eliminated in order that the lock is at the true center of the resonance line. One method of eliminating this undesirable signal is to introduce a signal of equal amplitude and opposite phase at some convenient point before the input of the lock-in amplifier.

The adjustment of this bucking signal is perhaps the most important one of all and should be checked carefully both above and below the resonance frequency before recording data. Since the oscillator frequency is changing as the NQR signal is tracked, the relative capacity of the tank circuit is also changing which means the bucking signal must be adjusted at each datum point to assure that the measurement is being made at the line center. When the $r$-f level is relatively high the change in level resulting from a change in frequency is quite small, since the feedback is small, and in this case it is possible to adjust the bucking voltage and phase to produce a relatively stable base line. At low r-f levels, where the feedback is large, the oscillator is most sensitive to the shunt impedance and changes in the relative capacity tend to cause base line drift. Hence the operating point must be such that the $r$-f level is large enough to ensure a stable base line over a frequency range comparable to the line width and yet small enough to avoid saturation of the nuclear resonance.

In the case of $\mathrm{KClO}_{3}$ we were able to select an operating point which gave the maximum signal-to-noise ratio and, with proper adjustment of the bucking voltage, a stable base line over the line width. In order to carry out the adjustment on the bucking signal it was necessary to unlock the spectrometer and manually shift the frequency, by adjusting the voltage on VCD1, to points on both sides of the resonance. Proper adjustment of the bucking voltage, as evidenced by the output of the lock-in detector, showed no noticeable change in the error voltage at points slightly above and slightly below resonance. The servo is then relocked to the center of the resonance and data recorded.

\section{System Performance}

The NQR spectrometer described in this paper was designed around the ${ }^{35} \mathrm{Cl}$ resonance in $\mathrm{KClO}_{3}$. As previously mentioned the operating frequency is $28 \mathrm{MHz}$. The servo system used here to track the NQR signal has a range of about 2.0 MHz. The magnitude of the tracking range is related to the change in capacity of the varicap VCD2, the coupling capacitor $C_{3}$, the amplifier bandwidth, and the frequency of operation. If $\Delta C$ is the effective change in capacity of the tank circuit, $f_{0}$ is the operating frequency, and $C_{0}$ is the total tank circuit capacity associated with $f_{0}$, then the frequency range is given by $\frac{\Delta f}{f}=$ $\frac{1}{2} \frac{\Delta C}{C_{0}}$ where the magnitude of $\Delta C$ is given by $\Delta C=$ $\frac{\Delta C_{v}}{\left(1+C_{v} / C_{3}\right)^{2}}$. Since $C_{v}$ and $\Delta C_{v}$ are fixed properties of the varicap and since $\Delta C_{v}$ depends only on the range of bias voltage, $\Delta f$ will be larger at the higher frequencies. In the case where $C_{v} / C_{3}<<1$ and $C^{\prime}<C_{v}$, where $C^{\prime}$ is the parallel tank circuit capacity excluding $C_{v}$ and $C_{3}$, nearly all of the current passing through $L$ will pass through $C_{v}$. This, coupled with the fact that the $\mathrm{r}$-f voltage across both $L$ and $C_{v}$ is nearly equal, causes the varicap to be the principle frequency controlling element. In addition, the major circuit losses occur in $L$ and $C_{v}$ and the varicap $Q$ becomes very important in determining the overall circuit $Q$. This becomes important at the higher frequencies. At the lower frequencies $C^{\prime}$ is larger than $C_{v}$ and the varicap $Q$ becomes less important.

Some care should be given to the selection of $C_{3}$. If $C_{3}$ is large compared to $C_{v}$ and the resonance line is narrow, small error signals applied to $C_{v}$ may result in frequency corrections larger than the line width thus causing the 
system to lose lock. On the other hand, if $C_{3}$ is small compared to $C_{v}$ the sensitivity of the servo is reduced.

After the spectrometer has been adjusted for maximum signal-to-noise and the bucking voltage properly set, the servo is locked to the center of the resonance line. In $\mathrm{KClO}_{3}$ the signal-to-noise is about 60:1 at room temperature and the line width [9] is about $500 \mathrm{~Hz}$. The phase on the lock-in detector is then adjusted for proper servo control. Depending on the signal to noise it may be necessary to readjust the gain and time constant to stabilize the servo loop gain.

In principle the servo amplifier is capable of tracking over its entire frequency range. In our experiment, however, a large sudden pressure change, corresponding to frequency increments of the order of $30 \mathrm{KHz}$, would cause the servo to become unlocked from the center of the resonance line, where as a gradual pressure change usually did not affect the lock. On the other hand, if the sample was near thermal equilibrium, the spectrometer, once locked to the resonance, would remain in the locked mode for several hours. The reason for the loss of lock is probably due to inhomogenous broadening of the resonance line associated with the temperature gradients at the sample resulting from the sudden change in pressure $\left(\left(\frac{\partial f}{\partial T}\right)_{P}=5 \mathrm{KHz} /{ }^{\circ} \mathrm{C}\right)$. In those cases where the servo amplifier became unlocked from the resonance signal it could easily be relocked manually to the center of the resonance line after a few minutes.

\section{Applications}

The NQR spectrometer described thus far could be adapted to any system in which perturbations on the system result in a shift of the NQR frequency. In the case where pressure is used as the perturbation many special problems must also be considered, namely, (1) a low loss feed-through, capable of withstanding high pressure is required in order to get the $r-f$ signal inside the pressure vessel, (2) due to the small working volume, sample size must be kept to a minimum, and (3) depending on temperature sensitivity of the sample, temperature control must be strictly maintained. The latter is perhaps the most difficult problem to deal with.

We have solved some of these problems with reasonable success. The pressure vessel is a commercially available unit [15] and the r-f leads are brought out through a high pressure electrical feedthrough similar to that described by Heydemann [16]. This arrangement has worked satisfactorily for pressures up to $2 \mathrm{kbar}$. The sample chamber in this pressure vessel is about $25 \mathrm{~mm}$ in diameter by $75-\mathrm{mm}$ long and our sample is a cylinder $5 \mathrm{~mm}$ in diameter and $10-\mathrm{mm}$ long. By keeping the coil winding diameter small compared to the diameter of the sample chamber the decrease in inductance due to shielding is kept to a minimum.

The temperature dependence of the NQR frequency of ${ }^{35} \mathrm{Cl}$ in $\mathrm{KClO}_{3}$ is considerably larger than the pressure dependence. For this reason we immersed the pressure vessel in a temperature-regulated oil bath. The temperature control was of the order of $\pm 0.015{ }^{\circ} \mathrm{C}$, measured in

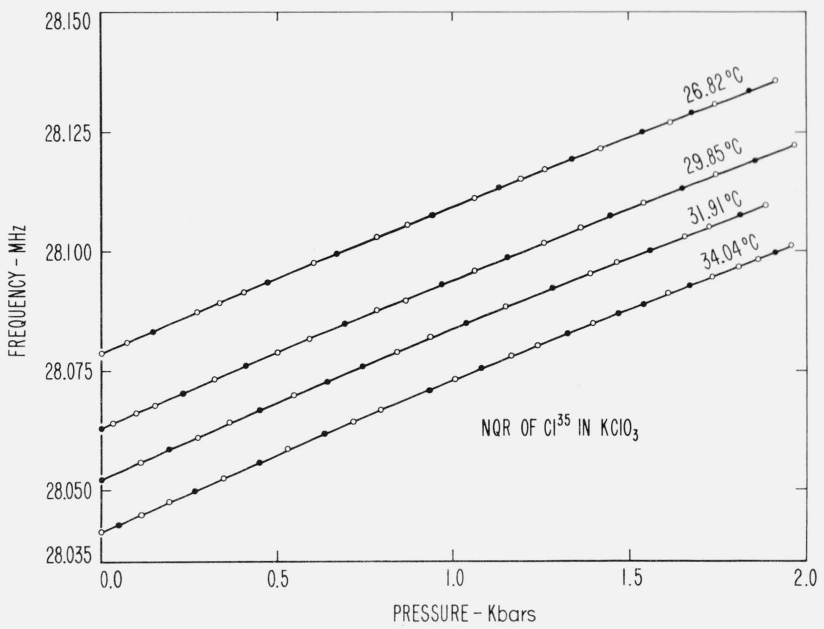

Figure 5. Plot of frequency-pressure-temperature data for ${ }^{35} \mathrm{Cl}$ in $\mathrm{KClO}_{3}$.

The temperature of each curve was determined by comparing the observed NQR frequency at zero pressure with the Spline fitting given by Utton [8]. Open circles indicate increasing values; solid circles, decreasing values.

the bath, over a 24-hr period corresponding to about 75 $\mathrm{Hz}$ or \pm 2.5 bars at room temperature.

The stability of the oscillator was determined using a Systron and Donner 1037 frequency counter which was calibrated against an NBS standard frequency. After a l-hr warm-up period the short term stability of this oscillator, when unlocked, is within $\pm 3 \mathrm{~Hz}$; the long-term stability is of the order of $\pm 100 \mathrm{~Hz}$. The stability of the spectrometer when locked to a resonance depends on the stability of the oscillator and on the sample temperature control. The short term stability in the locked mode, with the pressure vessel and sample immersed in the oil bath, is within $\pm 5 \mathrm{~Hz}$ where $5 \mathrm{~Hz}$ is the standard deviation of twenty l-s frequency counts. The long term stability in the locked mode is of the order of $\pm 4.5 \mathrm{~Hz}$, although this includes slight temperature drifts.

Samples were prepared by slow recrystallization to improve the sample purity as described by Utton [17]. A plot of the ${ }^{35} \mathrm{Cl}$ NQR frequency as a function of pressure is shown in figure 5 . The data shown here are the results of preliminary experiments for the purpose of determining the feasibility of using NQR as a pressure measuring device. The choice of $\mathrm{KClO}_{3}$ as a possible material for pressures less than 6 kbar stems from the good signal-tonoise ratio and narrow line width of the ${ }^{35} \mathrm{Cl}$ resonance, as well as a lack of frequency hysteresis with temperature [8]. $\mathrm{KClO}_{3}$ would not be an acceptable material for a larger pressure range due to a phase transition at $6 \mathrm{kbar}$ [18] at room temperature. The values used for pressure were recorded from a $0-40,000$ psi bourdon tube gage [19] and are believed to be accurate to $\pm 40 \mathrm{psi}$. The accuracy to which the NQR frequency can be recorded is $\pm 5 \mathrm{~Hz}$. However, due to the large temperature coefficient in $\mathrm{KClO}_{3}$, and the regulation of the oil bath, $\left( \pm .015{ }^{\circ} \mathrm{C}\right)$, the estimated uncertainty due to temperature instability is $\pm 75 \mathrm{~Hz}$. Hence the total estimated uncertainty in the frequency for a given pressure and temperature is $160 \mathrm{~Hz}$ or 5.3 bars. The value of tempera- 
ture assigned to each curve was determined by comparing the NQR frequency at zero pressure to the temperature data given by Utton [8]. As previously mentioned this type of temperature measurement is accurate to \pm 0.001 ${ }^{\circ} \mathrm{C}$. Since we were interested in small temperature changes, a thermopile consisting of four junctions was used. Fluctuations in temperature were then monitored based on the changes in thermopile EMF. At any temperature the data points, plotted in figure 5 , are from both increasing and decreasing pressures. The increasing pressure points are denoted by open circles and the decreasing points by solid circles. For each data point the value of the NQR frequency, pressure, and thermopile EMF, was recorded. The data points shown on the isotherms of figure 4 are normalized to the given temperature which means that although the real data points included small temperature variations, corrections of $4.90 \mathrm{KHz} /{ }^{\circ} \mathrm{C}$ were applied for adjustment; deviations between the line drawn and these values did not exceed $50 \mathrm{~Hz}$. Along the lines of constant pressure the temperature coefficient calculated from the data was found to be $4.93 \mathrm{KHz} /{ }^{\circ} \mathrm{C}$ below 6000 psi and $4.86 \mathrm{KHz} /{ }^{\circ} \mathrm{C}$ at the 2 kbar limit. Qualitatively, this difference agrees with the change in the temperature coefficient with pressure indicated by Benedek et al. [3]. Furthermore, the data shown here demonstrate that $\mathrm{KClO}_{3}$ exhibit no detectable hysteresis in this pressure range.

The results of our preliminary investigation clearly indicate that nuclear quadrupole resonance can be used as a pressure measuring technique. However, substantial progress towards establishing temperature-pressurefrequency curves will depend on more accurate pressure measurements and on controlling temperature more carefully. Experiments are presently in progress towards improving the temperature regulation of the oil bath to $\pm 0.001{ }^{\circ} \mathrm{C}$. We are also considering other materials which have a much smaller temperature coefficient than
$\mathrm{KClO}_{3}$. Improving the accuracy of the pressure measurement can only be accomplished by using a more accurate pressure standard such as a piston gage.

We thank Thomas C. Farrar, who suggested this problem, for many helpful discussions. We would also like to thank Peter Heydemann for the use of the pressure vessel and also for constructing the electrical feedthrough; and Daniel Buchalter for his assistance in the fabrication of the high pressure system.

\section{References}

[1] Bayer, H., Z. Physick 130, 227 (1951).

[2] Kushida, T., Benedek, G., and Bloembergen, N., Phys. Rev. 104, 1364 (1956).

[2a] Livingston, R., and Beldes, Mol. Phys. 3, 501-524, D. Williams, Ed. (1962).

[3] Benedek, G., and Kushida, T., Rev. Sci. Instr. 28, 92, (1957).

[4] Dean, C., and Pound, R. V., J. Chem. Phys. 20, 195 ( 1952 ).

[5] Vanier, J., Can. J. Phys. 38, 1397 (1960).

[6] Vanier, J., Metrologia 1, 135 (1965).

[7] Volpicelli, R. J., Nageswara Rao, B. D., and Baldeschwieler, J. D., Rev. Sci. Instr. 36, 150 (1965).

[8] Utton, D. B., International Journal of Scientific Metrology 3, No. 4, 98 (1967).

[9] Heydemann, Peter L. M., Private communication.

[10] Watkins, G., Thesis Howard University, 1952, Pound, R. V., and Knight, W. D., Rev. Sci. Instr. 21, 219 (1959).

[11] Robinson, F. N. H., J. Sci. Instr. 36, 481 (1959).

[12] Valley, G. E., Jr., Wallman, H., M.I.T. Radiation Laboratory Series 18, 166 ( 1964).

[13] Systron-Donner SD-1037B4D1F.

[14] Honeywell Amplifier \#361925.

[15] The pressure vessel was on loan from Dr. P. L. M. Heydemann.

[16] Heydemann, P. L. M., Rev. Sci. Instr. 38, 558 (1967).

[17] Utton, D. B., J. Res. Nat. Bur. Stand. (U.S.), 71A (Phys. and Chem.), No. 2, 125 (March-Apr. 1967).

[18] Bridgman, P. W., Proc. Am. Acad. Sci. 51, 55 (1915).

[19] Heise Bourdon Tube Co. Inc., Newtown, Conn.

(Paper 74C1\&2-293) 